\title{
The function of myostatin in the regulation of fat mass in mammals
}

\author{
Bing Deng ${ }^{1}$, Feng Zhang ${ }^{2}$, Jianghui Wen ${ }^{3}$, Shengqiang Ye ${ }^{1}$, Lixia Wang ${ }^{1}$, Yu Yang ${ }^{1}$, Ping Gong ${ }^{1}$ and Siwen Jiang ${ }^{2,4^{*}}$
}

\begin{abstract}
Myostatin (MSTN), also referred to as growth and differentiation factor-8, is a protein secreted in muscle tissues. Researchers believe that its primary function is in negatively regulating muscle because a mutation in its coding region can lead to the famous double muscle trait in cattle. Muscle and adipose tissue develop from the same mesenchymal stem cells, and researchers have found that MSTN is expressed in fat tissues and plays a key role in adipogenesis. Interestingly, MSTN can exert a dual function, either inhibiting or promoting adipogenesis, according to the situation. Due to its potential function in controlling body fat mass, MSTN has attracted the interest of researchers. In this review, we explore its function in regulating adipogenesis in mammals, including preadipocytes, multipotent stem cells and fat mass.
\end{abstract}

Keywords: Myostatin, Fat, Adipogenesis, Signaling pathway, Regulation

\section{Background}

Adipose tissues, which are mainly composed of adipocytes, play important roles in storage and metabolism $[1,2]$. The adipocytes in adipose tissues can be derived from mesenchymal stem cells with the appropriate stimulation. The differentiation process involves two phases: determination, in which multipotent stem cells become adipoblasts, and, differentiation, in which preadipocytes convert to mature adipocytes in the adipogenesis-promoting environment [2]. Myostatin (MSTN), a negative regulator of skeletal muscle growth, can be detected in not only muscle tissues but also adipose tissues [3]. Evidence has now been obtained demonstrating that MSTN could regulate the adipogenesis of mesenchymal stem cells in the determination and differentiation phases [4-6]. In this review, we mainly summarize the structure, tissue distribution, and signal transduction of MSTN and explore the role of MSTN in the adipogenesis of preadipocytes, multipotent stem cells and transgenic animals in mammals.

\footnotetext{
* Correspondence: jiangsiwen@mail.hzau.edu.cn

${ }^{2}$ Key Laboratory of Swine Genetics and Breeding of the Agricultural Ministry and Key Laboratory of Agricultural Animal Genetics, Breeding and Reproduction of the Ministry of Education, College of Animal Science and Technology, Huazhong Agricultural University, Wuhan 430070, People's Republic of China

${ }^{4}$ The Cooperative Innovation Center for Sustainable Pig Production, Wuhan 430070, China

Full list of author information is available at the end of the article
}

\section{The identification of MSTN and its inhibitory effect on muscle differentiation}

MSTN, also known as growth and differentiation factor-8, is mainly expressed in skeletal muscle and is a negative regulator of skeletal muscle growth in animals. It was initially identified in 1997 as a member of the transforming growth factor- $\beta$ superfamily using the degenerate polymerase chain reaction [3]. In the same year, the bovine MSTN gene was mapped to the mh locus by genetic linkage, which strongly suggested that MSTN may be the gene that causes double muscling in cattle [7]. This trait is useful in farm animals because it can dramatically increase muscular mass and improve economic benefits. Further analysis indicated that an 11-bp deletion in the coding region of MSTN in Belgian Blue cattle and a G-A missense mutation in the same region in Piedmontese cattle could cause the double-muscling trait in cattle [8].

Muscle progenitors and myoblast cells can proliferate and terminally differentiate into muscle fibers, which contribute to the growth of muscle mass [9]. The main functions of MSTN in muscle progenitors and myoblasts are self-renewal and differentiation inhibition. For example, in myoblasts, MSTN can inhibit myoblast differentiation into myotubes by preventing myogenic differentiation factor (MyoD) activity and expression via Smad $3[10,11]$. Similar findings have also been reported in mouse skeletal muscle $\mathrm{C} 2 \mathrm{C} 12$ cells, in which MSTN may control muscle 
mass by inhibiting cell proliferation and DNA and protein synthesis [12]. Further research in satellite cells shows that MSTN could negatively regulate satellite cell activation and control the self-renewal process of satellite cells [13].

\section{MSTN structure}

All transforming growth factor- $\beta$ (TGF- $\beta$ ) family members contain three distinct domains: an $\mathrm{N}$-terminal signal domain, a propeptide domain and a $\mathrm{C}$-terminal mature peptide $[14,15]$. As a member of the TGF- $\beta$ superfamily, MSTN shares the typical characteristics of other TGF- $\beta$ superfamily members: 1) a hydrophobic core of amino acids near the $\mathrm{N}$-terminus; 2) a conserved proteolytic processing signal of RSRR in the C-terminus; and 3 ) cysteine residues in the $\mathrm{C}$-terminal region to facilitate the formation of a "cysteine knot" structure [16-18]. The difference between MSTN and other TGF- $\beta$ superfamily members is that the nucleotide sequence of the $\mathrm{C}$-terminus is shorter than those in other members [3].

\section{MSTN tissue expression}

MSTN can not only be detected in muscle but can also exert its function in other tissues. Initial research studies in 1997 found that MSTN is predominantly expressed in the muscle tissues of mice and cows $[3,8]$, but it is also detected in the adipose tissue [3]. Further research showed that MSTN could be detected in mammary glands [19], Purkinje fibers and cardiomyocytes in heart tissue [20], spleen, lymphocytes [21], placenta [22], and even in human uterine gland muscle tissue [23]. Those results indicated that MSTN may exert its function not only in muscle but also in other tissues.

\section{The function of MSTN in fat formation in mammals}

The results of many past studies have indicated that MSTN plays key roles in not only myogenesis but also adipogenesis. The function of MSTN in adipogenesis is controversial. In preadipocytes, MSTN mainly inhibits adipogenesis, whereas it can promote the adipogenesis of pluripotent stem cells. MSTN deletion and inhibition in animals mainly lead to increased muscle mass and reduced fat mass. Specific inhibition of MSTN in muscle but not adipose tissue inhibits fat mass. Specific overexpression of MSTN in adipose tissue increases the metabolic rate and resistance to diet-induced obesity. In the following section, we will explore the role of MSTN in fat formation in mammals.

\section{Differentiation is inhibited by MSTN in preadipocytes}

In different species of preadipocytes, MSTN mainly inhibits cell differentiation. For example, in 3T3-L1 preadipocytes treated with MSTN during differentiation, adipogenesis was significantly inhibited through the regulation of CCAAT/enhancer binding protein (C/EBP) $\beta$ and peroxisome proliferator-activated receptor $\gamma$ (PPARY) [24]. In addition, another adipogenesis transcription factor, $C / E B P a$, and lipid metabolism-related genes such as glycerol-3-phosphate dehydrogenase $(G P D H)$, diacylglycerol O-acyltransferase (DGAT), acylCoA synthetase long-chain family member1 (ACS1), adipose triglyceride lipase $(A T G L)$, and hormone-sensitive lipase $(H S L)$ are inhibited by MSTN in 3T3-L1 preadipocyte adipose differentiation [25]. Moreover, the adipogenesis of primary preadipocytes isolated from bovine, porcine fat tissue and intramuscular preadipocytes isolated from porcine longissimus dorsi muscles is also inhibited by treatment with adipogenesis medium plus MSTN [6, 26, 27].

The inhibition of brown adipogenesis by MSTN

White and brown adipocytes are two types of distinct adipocytes in mammals. White adipocytes mainly store excess energy in large lipid droplet, whereas brown adipocytes contain numerous smaller droplets and burn energy by non-shivering thermogenesis $[2,28]$. Research initially focused on the inhibition of adipogenesis by MSTN in white adipocytes. More recent research has revealed that MSTN can also inhibit the differentiation of brown preadipocytes. This process involves TGF- $\beta$ / Smad3 signaling [29] and Smad3-mediated $\beta$-catenin stabilization [30].

Mouse embryonic fibroblasts can differentiate into brown adipose-like cells under specialized adipogenic conditions. Primary mouse embryonic fibroblasts isolated from MSTN-deficient mice exhibit brown adipose-like differentiation and increased lipid metabolism and energy expenditure under specialized brown adipogenic conditions [31]. MSTN treatment in differentiating MSTN-deficient MEF can inhibit key BAT markers (Uncoupling Protein 1 (Ucp1) and PR domain containing 16 (Prdm16)) expression [32, 33]. These results indicate that MSTN can influence brown adipogenesis in mouse embryonic fibroblasts.

\section{The adipogenesis of pluripotent stem cells is promoted by MSTN}

C3H10T(1/2) cells, a mesenchymal fibroblast-like cell line of embryonic origin, have the capacity to undergo differentiation into multiple cell lineages, such as myoblasts, chondrocytes, and adipocytes, after being incubated in different media in vitro $[4,34,35]$. The potential for myogenic differentiation could be inhibited by MSTN, and MSTN can promote the commitment and differentiation of mesenchymal cells into the adipogenic lineages [34].

The function of MSTN in C3H10T(1/2) cell adipogenesis appears to involve driving the cells into a particular state. When cells are induced into adipocytes, DIM, 
which includes dexamethasone, insulin and isobutyl1-methylxanthine (IBMX), is always used to trigger adipogenesis [4]. The key component of adipogenesisinducing medium, dexamethasone, could induce MSTN expression [4, 36]. Pantoja et al. [37] demonstrated that treating $\mathrm{C} 3 \mathrm{H} 10 \mathrm{~T}(1 / 2)$ cells with dexamethasone for $48 \mathrm{~h}$ followed by IBMX treatment for $48 \mathrm{~h}$ was sufficient for adipogenesis, significant differentiation did not occur when $\mathrm{C} 3 \mathrm{H} 10 \mathrm{~T}(1 / 2)$ cells were treated with IBMX followed by dexamethasone. Moreover, recombinant MSTN could substitute dexamethasone in the DIM mixture to induce significant levels of adipogenesis in $\mathrm{C} 3 \mathrm{H} 10 \mathrm{~T}(1 / 2)$ cells, but not in 3T3-L1 cells (a preadipocyte cell line) [4]. Together, these data show that MSTN may induce adipogenesis in very-early-stage mesenchymal stem cells [4]. This special early stage needs to be clearly confirmed in further studies.

\section{MSTN deletion mainly leads to increased muscle mass, reduced fat mass and resistance to diet-induced obesity}

Muscle and adipose tissue develop from the same mesenchymal stem cells [2]. MSTN gene function seems to control the switch between adipogenesis and myogenesis. In a mouse model, Lin et al. [38] showed that MSTN knockout (KO) led to reduced adipogenesis and consequently decreased leptin secretion, which is associated with increased muscle development. Guo et al. [39] also showed that MSTN KO mice exhibited a dramatic increase in muscle mass and reduced fat mass but no changes in the whole-body lipid oxidation rate. By contrast, glucose utilization and insulin sensitivity increased in MSTN KO mice. In aging mice, the body fat percentage was also lower in MSTN KO compared with WT [40]. Decreased fat accumulation and increased muscle mass were also observed in MSTN KO rats [41] and pigs compared with wild type animals [42]. Previous research indicated that adipocytes and myocytes are both derived from the same mesodermal precursor [2]. The diminished fat mass and enhanced muscle in MSTN KO mice may be due to rapid depletion of the pool of stem, transit amplifying and progenitor (STP) cells in white adipose tissue (WAT) and brown adipose tissue (BAT) [43].

In addition, the MSTN KO mice also exhibited resistance to diet-induced obesity [39]. This resistance phenotype may be due to the transformation of white adipocytes to brown adipocytes. Zhang et al. [44] demonstrated that MSTN KO mice are resistant to high-fat diet-induced obesity via an increase in fatty acid oxidation in peripheral tissues and enhanced brown adipose formation in white adipose tissue. Further research indicated that MSTN KO mice can drive white adipose tissue into brown adipose tissue with the expression of BAT signature genes, including Ucp1 and peroxisomal proliferator-activated receptor coactivator 1 (Pgc1), and the beige adipocyte markers transmembrane protein 26 (Tmem26) and tumor necrosis factor receptor superfamily member 9 (TNFRSF9, CD137) by activating the AMPK-PGC1-Fndc5 pathway in muscle [32]. miR-34a is also involved in regulating fibronectin type III domain-containing protein (Fndc5) expression in active browning of white adipocytes [45].

\section{MSTN inhibition also leads to decreased fat tissue in mammals}

MSTN inhibition in animals has been investigated and could lead to decreased amounts of fat tissue. When MSTN was suppressed by a propeptide cDNA sequence in transgenic mice, the fat masses in the subcutaneous, epididymal and retroperitoneal areas were significantly less than in WT mice [46]. Similarly, visceral fat was decreased in adult mice upon knockdown of MSTN by siRNA [47]. McPherron et al. [48] found that MSTN inhibition may be more efficacious in reducing adipose weight gain rather than in causing weight loss when MSTN is inhibited by treatment with a soluble MSTN receptor derived from the activin receptor type IIB extracellular domain in high-fat diet-induced mice. Furthermore, the diet-induced obese rats showed reduced body and fat weight using a prepared polyclonal antibody for MSTN [49] and the myostatin antagonist sActRIIB [44]. Dong et al. [50] demonstrated that white adipose tissue is converted to brown adipose tissue and fatty acid oxidation and energy expenditure are promoted when myostatin is inhibited by an anti-myostatin peptibody in HFD-fed mice. This related mechanism is due to muscle-to-fat cross talk by Fndc5 (irisin).

\section{Tissue-specific MSTN inhibition and over-expression in mice}

The inhibition of myostatin signaling in adipose tissue had no effect on body composition in mice fed a standard diet or high-fat diet. By contrast, the inhibition of MSTN signaling in skeletal muscle increased lean mass and decreased fat mass on standard and high-fat diets, as well as resistance to diet-induced obesity [39]. The results indicated that specific inhibition of MSTN in skeletal muscle but not fat tissues can increase resistance to diet-induced obesity.

Adipose tissue-specific MSTN overexpression also increases resistance to diet-induced obesity. Feldman et al. [4] generated aP2-MSTN transgenic mice that express MSTN in fat tissue under the control of the aP2 promoter. The aP2-MSTN transgenic mice exhibited an increased metabolic rate and were resistant to diet-induced obesity. In addition, adipocytes induced by MSTN in both C3h10T1/2 cells and transgenic mice were small and expressed markers characteristic of immature adipocytes. 


\section{MSTN signal transduction in adipogenesis}

MSTN, a secreted protein, needs to transmit its signaling into the nucleus via a series cascade reaction to exert its function. Rebbapragada et al. [51] found that MSTN could first bind the type II Ser/Thr kinase receptor (ActRIIB) and then partner with a type I receptor, either activin receptor-like kinase 4 (ALK4 or ActRIB) or ALK5 (T $\beta R I)$, to induce $S \operatorname{mad} 2 / 3$ phosphorylation to inhibit the adipogenesis of C3H10T1/2 cells. Further research indicated that MSTN can activate Smad3 and the crosscommunication of the TGF- $\beta /$ Smad signal to the Wnt $\beta$ catenin/TCF4 pathway to down-regulate PPAR $\gamma$, leading to the inhibition of adipogenesis in human bone marrowderived mesenchymal stem cells and preadipocytes [52]. Recent research indicated that MSTN could regulate MyoD expression to influence PPAR $\gamma$ to exert its function in adipogenesis $[53,54]$.

\section{The regulation of MSTN gene expression}

As an important regulatory element in $5^{\prime}$ upstream regions of genes, the promoter can be bound by transcription factors to regulate gene expression. The analysis of the MSTN promoter shows that it can be regulated by many transcriptional factors. Li indicated that MSTN promoter activity is regulated by myocyte enhancer factor 2 in pigs [55]. In cattle and sheep, MSTN is regulated by the muscle-related transcription factor myogenic factor 5 (Myf5), myocyte enhancer factor-2 (MEF2) and MyoD [18, 56]. Deng et al. [27] showed that porcine MSTN could be upregulated by IBMX, MyoD and PPAR $\gamma$ but down-regulated by $\mathrm{C} / \mathrm{EBP} \alpha$ and $\mathrm{C} / \mathrm{EBP} \beta$ by analyzing the promoter of the porcine MSTN gene. In addition, MSTN could be induced by dexamethasone [4, 27]. Those studies indicated that MSTN can be regulated not only by myogenesis-related factors but also by adipogenesis factors.

\section{Conclusion}

As a factor involved in muscle and fat regulation, the function of MSTN in muscle has been widely investigated. In addition, its role in regulating fat mass has also attracted researchers' interest. It is clear that MSTN has positive and negative effects on adipogenesis depending on the situation. In preadipocytes, MSTN mainly inhibits adipogenesis $[23,26]$, while it promotes adipogenesis in pluripotent stem cells [4, 37]. Further studies utilizing MSTN transgenic animal models indicated that it mainly promotes fat mass accumulation. For example, MSTN gene knockout in animals mainly leads to reduced fat mass and resistance to diet-induced obesity $[39,41]$. MSTN inhibition in animals leads to reductions in fat tissues $[46,48,49]$. However, myostatin inhibition in muscle but not adipose tissue inhibits fat mass and improves insulin sensitivity [39]. Adipose tissue-specific
MSTN overexpression increases the metabolic rate and resistance to diet-induced obesity [4].

Although many reports show that MSTN participates in the regulation of adipogenesis, more details remain to be elucidated: 1) How does MSTN play different roles in preadipocytes and pluripotent stem cells? 2) To which cellular state does MSTN drive cells? 3) How does MSTN cross-communicate with other adipogenesis-related signaling factors, such as the Wnt signaling pathway?

A better understanding of MSTN in adipogenes is likely to be a novel and promising area for better clinical applications for controlling body and fat weight and animal production.

\begin{abstract}
Abbreviations
ACS 1: acyl-CoA synthetase long-chain family member 1; ActRIIB: Type II Ser/Thr kinase receptor; ALK4: Activin receptor-like kinase 4; ATGL: Adipose triglyceride lipase; BAT: Brown adipose tissue; C/EBP: CCAAT/enhancer binding protein; DGAT: Diacylglycerol O-acyltransferase; Fndc5: Fibronectin type III domain-containing protein; GPDH: Glycerol-3-phosphate dehydrogenase; HSL: Hormone-sensitive lipase; KO: Knockout; MEF2: Myocyte enhancer factor-2; MSTN: Myostatin; Myf5: Myogenic factor 5; MyoD: Myogenic differentiation factor; Pgc1: Peroxisomal proliferator-activated receptor coactivator 1; PPARy: Peroxisome proliferator-activated receptor $\gamma$; Prdm16: PR domain containing 16; TGF- $\beta$ : Transforming growth factor- $\beta$; Tmem26: Transmembrane protein 26; TNFRSF9, CD137: Tumor necrosis factor receptor superfamily member 9; Ucp1: Uncoupling Protein 1; WAT: White adipose tissue; WT: Wild-type
\end{abstract}

\section{Acknowledgments}

Not applicable.

Funding

This research was supported by the National Natural Science Foundation of China (31201766, 31472075, 31272425), the National Science \& Technology Pillar Program during the Twelfth Five-year Plan Period (2014BAD20B01, 2011BAD28B01), and the Science and Technology Planning Project of Hubei Province, China (2014ABA025).

Availability of data and materials

All data are available by direct request from the author.

Authors' contributions

$\mathrm{BD}$ and SJ participated in the design and writing of the review and are responsible for the final content of the manuscript; FZ, JW, SY, LW, YY, and PG helped collect the related literature; all authors read and approved the final manuscript.

\section{Competing interests}

The authors declare that they have no competing interests.

Consent for publication

Not applicable.

Ethics approval and consent to participate

As a review paper, no ethical approval was sought or required.

\section{Author details}

${ }^{1}$ Institute of Animal Husbandry and Veterinary Science, Wuhan Academy of Agricultural Science and Technology, Wuhan, Hubei 430208, People's Republic of China. ${ }^{2}$ Key Laboratory of Swine Genetics and Breeding of the Agricultural Ministry and Key Laboratory of Agricultural Animal Genetics, Breeding and Reproduction of the Ministry of Education, College of Animal Science and Technology, Huazhong Agricultural University, Wuhan 430070, People's Republic of China. ${ }^{3}$ Wuhan University of Technology, Wuhan 430074, People's Republic of China. ${ }^{4}$ The Cooperative Innovation Center for Sustainable Pig Production, Wuhan 430070, China. 
Received: 2 November 2016 Accepted: 24 February 2017 Published online: 21 March 2017

\section{References}

1. Gerbens F, Pas MFW, Everts ME, Haagsman HP. Genetic control of intramuscular fat accretion. In: te Pas MFW, Everts ME, Haagsman HP, editors. Muscle development of livestock animals: physiology, genetics and meat quality. 1st ed. Wallingford: CABI Publishing; 2004. p. 343-62.

2. Algire C, Medrikova D, Herzig S. White and brown adipose stem cells: from signaling to clinical implications. Biochim Biophys Acta. 2013;1831:896-904

3. McPherron AC, Lee SJ. Double muscling in cattle due to mutations in the myostatin gene. Proc Natl Acad Sci U S A. 1997;94:12457-61.

4. Feldman BJ, Streeper RS, Farese Jr RV, Yamamoto KR. Myostatin modulates adipogenesis to generate adipocytes with favorable metabolic effects. Proc Natl Acad Sci U S A. 2006;103:15675-80.

5. Deng B, Wen J, Ding Y, Gao Q, Huang H, Ran Z, et al. Functional analysis of pig myostatin gene promoter with some adipogenesis- and myogenesisrelated factors. Mol Cell Biochem. 2012:363:291-9.

6. Sun WX, Dodson MV, Jiang ZH, Yu SG, Chu WW, Chen J. Myostatin inhibits porcine intramuscular preadipocyte differentiation in vitro. Domest Anim Endocrinol. 2016;55:25-31.

7. Smith TPL, Lopez-Corrales NL, Kappes SM, Sonstegard TS. Myostatin maps to the interval containing the bovine mh locus. Mamm Genome. 1997:8:742-4.

8. Kambadur R, Sharma M, Smith TP, Bass JJ. Mutations in myostatin (GDF8) in double-muscled Belgian Blue and Piedmontese cattle. Genome Res. 1997;7:910-6.

9. Manceau M, Gros J, Savage K, Thomé V, McPherron A, Paterson B, et al. Myostatin promotes the terminal differentiation of embryonic muscle progenitors. Genes Dev. 2008;22:668-81.

10. Langley B, Thomas M, Bishop A, Sharma M, Gilmour S, Kambadur R. Myostatin inhibits myoblast differentiation by down-regulating MyoD expression. J Biol Chem. 2002;277:49831-40.

11. Amthor H, McKinnell I, Patel K. Stop and go - myostatin reversibly inhibits proliferation and differentiation of muscle precursors. Neuromuscul Disord. 2003;13:636

12. Taylor WE, Bhasin S, Artaza J, Byhower F, Azam M, Willard DH, et al. Myostatin inhibits cell proliferation and protein synthesis in C2C12 muscle cells. Am J Physiol Endocrinol Metab. 2001;280:E221-8.

13. McFarlane C, Hennebry A, Thomas M, Plummer E, Ling N, Sharma M, et al. Myostatin signals through $\mathrm{Pax} 7$ to regulate satellite cell self-renewal. Exp Cell Res. 2008;314:317-29.

14. Gordon KJ, Blobe GC. Role of transforming growth factor- $\beta$ superfamily signaling pathways in human disease. Biochim Biophys Acta. 2008;1782:197-228.

15. Siegel PM, Massagué J. Cytostatic and apoptotic actions of TGF-beta in homeostasis and cancer. Nat Rev Cancer. 2003;3:807-21.

16. McPherron AC, Lee S. Growth factors cytokines health. DIS. 1996;1B:357-93.

17. Thomas M, Langley B, Berry C, Sharma M, Kirk S, Bass J, et al. Myostatin, a negative regulator of muscle growth, functions by inhibiting myoblast proliferation. J Biol Chem. 2000;275:40235-43.

18. Spiller MP, Kambadur R, Jeanplong F, Thomas M, Martyn JK, Bass JJ, et al. The myostatin gene is a downstream target gene of basic helix-loop-helix transcription factor MyoD. Mol Cell Biol. 2002;22:7066-82.

19. Ji S, Losinski RL, Cornelius SG, Frank GR, Willis GM, Gerrard DE, et al. Myostatin expression in porcine tissues: tissue specificity and developmental and postnatal regulation. Am J Physiol. 1998;275:R1265-73.

20. Sharma M, Kambadur R, Matthews KG, Somers WG, Devlin GP, Conaglen JV, et al. Myostatin, a transforming growth factor-beta superfamily member, is expressed in heart muscle and is upregulated in cardiomyocytes after infarct. J Cell Physiol. 1999;180:1-9.

21. Lyons JA, Haring JS, Biga PR. Myostatin expression, lymphocyte population, and potential cytokine production correlate with predisposition to high-fat diet induced obesity in mice. PLoS One. 2010;5:e12928.

22. Mitchell MD, Osepchook CC, Leung KC, McMahon CD, Bass JJ. Myostatin is a human placental product that regulates glucose uptake. J Clin Endocrinol Metab. 2006:91:1434-7.

23. Carrarelli $P$, Yen CF, Arcuri F, Funghi L, Tosti C, Wang TH, et al. Myostatin, follistatin and activin type II receptors are highly expressed in adenomyosis. Fertil Steril. 2015;104:744-52. e1.
24. Kim HS, Liang L, Dean RG, Hausman DB, Hartzell DL, Baile CA. Inhibition of preadipocyte differentiation by myostatin treatment in 3T3-L1 cultures. Biochem Biophys Res Commun. 2001;281:902-6.

25. Zhu HJ, Pan H, Zhang XZ, Li NS, Wang LJ, Yang HB, et al. The effect of myostatin on proliferation and lipid accumulation in 3T3-L1 preadipocytes. J Mol Endocrinol. 2015:54:217-26.

26. Hirai S, Matsumoto $H$, Hino N, Kawachi H, Matsui T, Yano H. Myostatin inhibits differentiation of bovine preadipocyte. Domest Anim Endocrinol. 2007:32:1-14

27. Deng B, Wen J, Ding Y, Peng J, Jiang S. Different regulation role of myostatin in differentiating pig ADSCs and MSCs into adipocytes. Cell Biochem Funct. 2012;30:145-50.

28. Virtanen KA. BAT thermogenesis: linking shivering to exercise. Cell Metab. 2014; 19:352-4

29. Fournier B, Murray B, Gutzwiller S, Marcaletti S, Marcellin D, Bergling S, et al. Blockade of the activin receptor Ilb activates functional brown adipogenesis and thermogenesis by inducing mitochondrial oxidative metabolism. Mol Cell Biol. 2012;32:2871-9.

30. Kim WK, Choi HR, Park SG, Ko Y, Bae KH, Lee SC. Myostatin inhibits brown adipocyte differentiation via regulation of Smad3- mediated $\beta$-catenin stabilization. Int J Biochem Cell Biol. 2012:44:327-34.

31. Braga M, Pervin S, Norris K, Bhasin S, Singh R. Inhibitionof in vitro and in vivo brownfatdifferentiationprogrambymyostatin. Obesity. 2013;21:1180-8.

32. Shan $T$, Liang $X, B i P$, Kuang $S$. Myostatin knockout drives browning of white adipose tissue through activating the AMPK-PGC1a-Fndc5 pathway in muscle. FASEB J. 2013;27:1981-9.

33. Singh $R$, Braga M, Pervin S. Regulation of brown adipocyte metabolism by myostatin/follistatin signaling. Front Cell Dev Biol. 2014;2:60.

34. Artaza JN, Bhasin S, Magee TR, Reisz-Porszasz S, Shen R, Groome NP, et al. Myostatin inhibits myogenesis and promotes adipogenesis in $\mathrm{C} \mathrm{H}_{10} \mathrm{~T}_{(1 / 2)}$ mesenchymal multipotent cells. Endocrinology. 2005;146:3547-57.

35. Muruganandan S, Roman AA, Sinal CJ. Adipocyte differentiation of bone marrow-derived mesenchymal stem cells: cross talk with the osteoblastogenic program. Cell Mol Life Sci. 2009:66:236-53.

36. Ma K, Mallidis C, Bhasin S, Mahabadi V, Artaza J, Gonzalez-Cadavid N, et al Glucocorticoid-induced skeletal muscle atrophy is associated with upregulation of myostatin gene expression. Am J Physiol Endocrinol Metab. 2003;285:E363-71.

37. Pantoja C, Huff JT, Yamamoto KR. Glucocorticoid signaling defines a novel commitment state during adipogenesis in vitro. Mol Biol Cell. 2008;19:4032-41.

38. Lin J, Arnold HB, Della-Fera MA, Azain MJ, Hartzell DL, Baile CA. Myostatin knockout in mice increases myogenesis and decreases adipogenesis. Biochem Biophys Res Commun. 2002;291:701-6.

39. Guo T, Jou W, Chanturiya T, Portas J, Gavrilova O, McPherron AC. Myostatin inhibition in muscle, but not adipose tissue, decreases fat mass and improves insulin sensitivity. PLoS One. 2009;4:e4937.

40. Morissette MR, Stricker JC, Rosenberg MA, Buranasombati C, Levitan EB, Mittleman MA, et al. Effects of myostatin deletion in aging mice. Aging Cell. 2009;8:573-83.

41. Gu H, Cao Y, Qiu B, Zhou Z, Deng R, Chen Z, et al. Establishment and phenotypic analysis of an Mstn knockout rat. Biochem Biophys Res Commun. 2016;477:115-22

42. Qian L, Tang M, Yang J, Wang Q, Cai C, Jiang S, et al. Targeted mutations in myostatin by zinc-finger nucleases result in double-muscled phenotype in Meishan pigs. Sci Rep. 2015:5:14435.

43. Li N, Yang Q, Walker RG, Thompson TB, Du M, Rodgers BD. Myostatin attenuation in vivo reduces adiposity, but activates adipogenesis. Endocrinology. 2016;157:282-91.

44. Zhang C, McFarlane C, Lokireddy S, Masuda S, Ge X, Gluckman PD, et al. Inhibition of myostatin protects against diet-induced obesity by enhancing fatty acid oxidation and promoting a brown adipose phenotype in mice. Diabetologia. 2012;55:183-93.

45. Ge X, Sathiakumar D, Lua BJ, Kukreti H, Lee M, McFarlane C. Myostatin signals through miR-34a to regulate Fndc5 expression and browning of white adipocytes. Int J Obes (Lond). 2017;41:137-48.

46. Zhao B, Wall RJ, Yang J. Transgenic expression of myostatin propeptide prevents diet-induced obesity and insulin resistance33. Biochem Biophys Res Commun. 2005:337:248-55.

47. Mosler S, Relizani K, Mouisel E, Amthor H, Diel P. Combinatory effects of siRNA-induced myostatin inhibition and exercise on skeletal muscle homeostasis and body composition. Physiol Rep. 2014;2:e00262. 
48. McPherron AC, Guo T, Wang Q, Portas J. Soluble activin receptor type IIB treatment does not cause fat loss in mice with diet-induced obesity. Diabetes Obes Metab. 2012;14:279-82.

49. Tang L, Yang X, Gao X, Du H, Han Y, Zhang D, et al. Inhibiting myostatin signaling prevents femoral trabecular bone loss and microarchitecture deterioration in diet-induced obese rats. Exp Biol Med (Maywood). 2016;241:308-16.

50. Dong J, Dong Y, Dong Y, Chen F, Mitch WE, Zhang L. Inhibition of myostatin in mice improves insulin sensitivity via irisin-mediated cross talk between muscle and adipose tissues. Int J Obes (Lond). 2016;40:434-42.

51. Rebbapragada A, Benchabane H, Wrana JL, Celeste AJ, Attisano L. Myostatin signals through a transforming growth factor beta-like signaling pathway to block adipogenesis. Mol Cell Biol. 2003;23:7230-42.

52. Guo W, Flanagan J, Jasuja R, Kirkland J, Jiang L, Bhasin S. The effects of myostatin on adipogenic differentiation of human bone marrow-derived mesenchymal stem cells are mediated through cross-communication between Smad3 and Wnt/beta-catenin signaling pathways. J Biol Chem. 2008;283:9136-45.

53. Zhang F, Deng B, Wen J, Chen K, Liu W, Ye S, et al. PPARy and MyoD are differentially regulated by myostatin in adipose-derived stem cells and muscle satellite cells. Biochem Biophys Res Commun. 2015;458:375-80.

54. Deng B, Zhang F, Chen K, Wen J, Huang H, Liu W, et al. MyoD promotes porcine PPARy gene expression through an E-box and a MyoD-binding site in the PPARy promoter region. Cell Tissue Res. 2016;365:381-91.

55. Li J, Deng J, Zhang J, Cheng D, Wang H. Regulation of myostatin promoter activity by myocyte enhancer factor. Sheng Wu Gong Cheng Xue Bao. 2012;28(8):918-26.

56. Du R, An X, Chen Y, Qin J. Functional analysis of the myostatin gene promoter in sheep. Sci China C Life Sci. 2007;50:648-54.

\section{Submit your next manuscript to BioMed Central and we will help you at every step:}

- We accept pre-submission inquiries

- Our selector tool helps you to find the most relevant journal

- We provide round the clock customer support

- Convenient online submission

- Thorough peer review

- Inclusion in PubMed and all major indexing services

- Maximum visibility for your research

Submit your manuscript at www.biomedcentral.com/submit 